
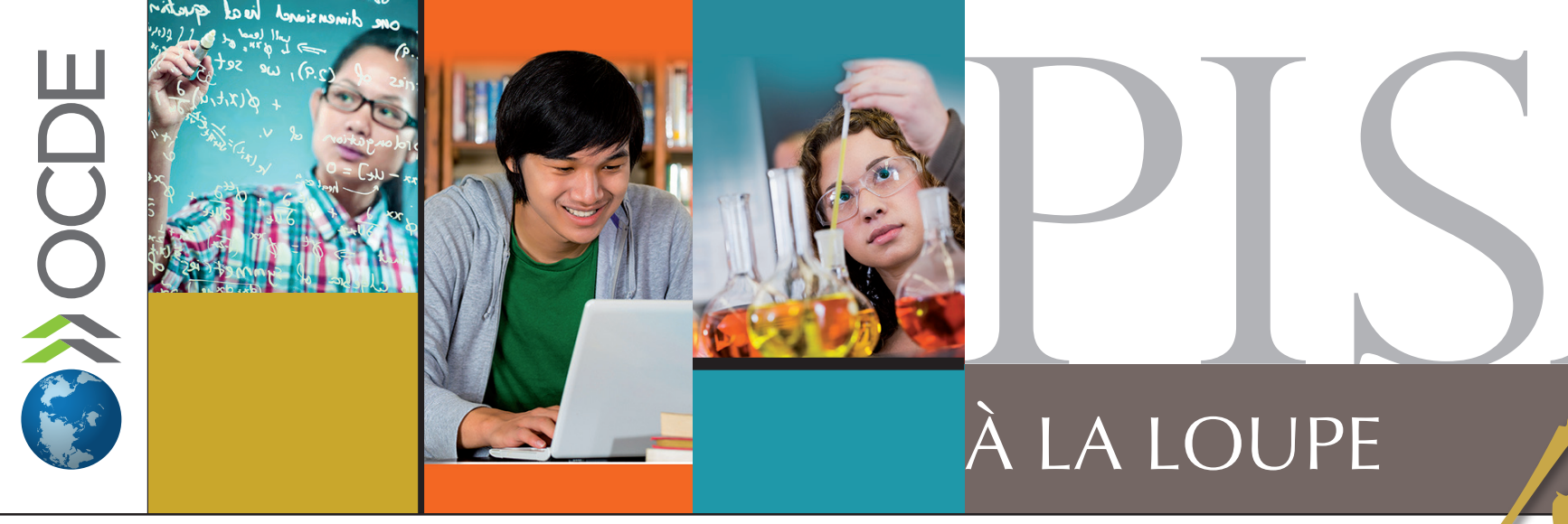

éducation données éducation chiffres éducation politiques éducation analyses éducation statistiques éducation donnée, éd

\title{
Comment la performance des élèves a-t-elle évolué au fil du temps?
}

- L'amélioration de la performance d'un pays dans l'enquête PISA n'est liée ni à sa situation géographique, ni à son niveau de richesse nationale, ni à sa culture.

- Dans la plupart des cas, les pays qui ont enregistré une amélioration marquée de leur performance dans l'enquête PISA - à savoir l'Allemagne, le Brésil, la Grèce, l'Italie, le Mexique, la Tunisie et la Turquie - sont ceux qui sont parvenus à réduire leur pourcentage d'élèves peu performants.

- Même au fil du temps, l'excellence et l'équité ne constituent pas deux objectifs incompatibles, comme en attestent les progrès observés en Allemagne, en Italie, au Mexique, en Tunisie et en Turquie.

Tous les trois ans, lors de la publication des résultats de l'enquête PISA, les médias du monde entier braquent leurs projecteurs sur les classements des pays en mathématiques, en compréhension de l'écrit et en sciences. Or souvent, l'examen de conscience national qui s'ensuit pour réfléchir aux moyens d'améliorer la performance des élèves fait oublier que de nombreux pays ont sensiblement relevé leur niveau depuis la première enquête PISA en 2000. Ainsi, la moitié des pays et économies ayant participé à au moins trois enquêtes PISA ont vu leurs résultats s'améliorer de façon sensible en compréhension de l'écrit depuis 2000, un tiers, en mathématiques depuis 2003, et près d'un tiers, en sciences depuis 2006.

Tous les pays/économies peuvent s'améliorer et même assez rapidement.

L'amélioration de la performance d'un pays dans l'enquête PISA n'est pas tributaire de sa situation géographique, de son niveau de richesse nationale ou de son patrimoine culturel. Ainsi, Singapour, petit pays $\mathrm{d}^{\prime}$ Asie relativement riche (et deuxième du classement de la performance en mathématiques de l'enquête PISA 2012), a vu son score augmenter d'environ 4 points par an, tout comme le Brésil, vaste pays d'Amérique latine à revenu intermédiaire, où deux élèves sur trois n'atteignent toujours pas le niveau de compétence de base en mathématiques (soit le niveau 2). Des pays aussi différents que l'Allemagne, le Chili, Israël, la Malaisie, le Qatar et la Roumanie ont également vu leur performance en mathématiques s'améliorer sensiblement. 


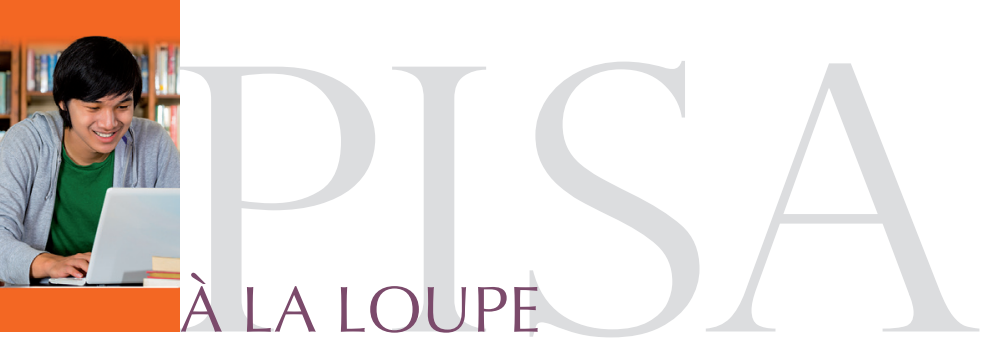

Au fil des années, il ressort des résultats des enquêtes PISA que le changement peut s'opérer relativement vite - bien plus vite en tout cas que ce que I'on pensait auparavant. Ainsi, en tout juste dix ans, la Pologne a vu son score en mathématiques passer de 490 à 518 points, d'un niveau inférieur à la moyenne de l'OCDE à un niveau largement supérieur à cette dernière. Son score en compréhension de l'écrit est quant à lui passé de 479 à 518 points - soit une différence équivalant à une année entière de scolarité. Cette amélioration est en partie liée à la réforme structurelle lancée par la Pologne en 1999. En outre, le Brésil, la Bulgarie, Israël, I'Italie, le Mexique, le Portugal, le Qatar, la Roumanie, la Serbie, la Tunisie et la Turquie, qui ont tous participé à au moins trois enquêtes PISA, ont vu leur performance en mathématiques augmenter d'au moins 2 points de score par an depuis 2003.

\section{Pays ayant connu une évolution sensible de leur performance en mathématiques entre 2003 et 2012}

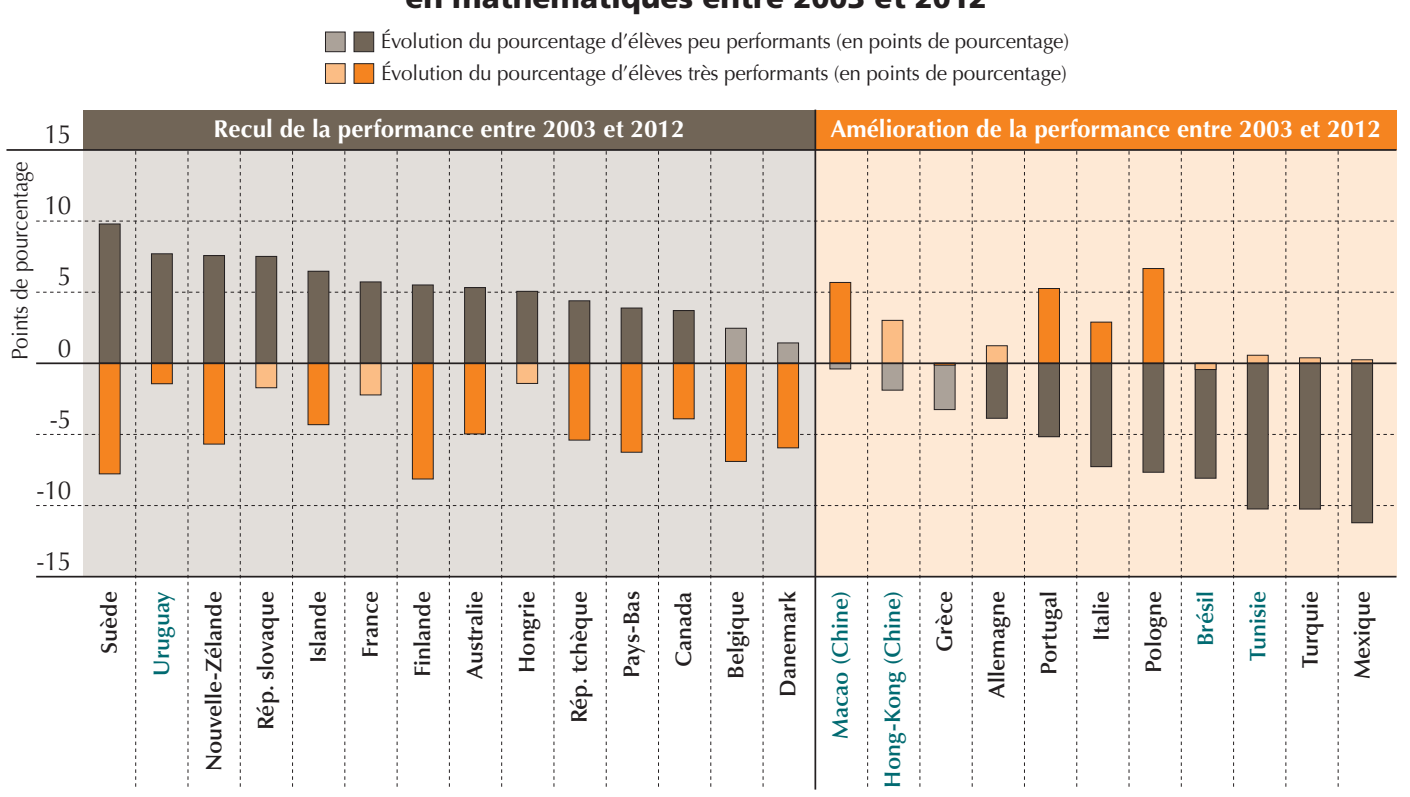

Remarque : les évolutions statistiquement significatives sont indiquées dans une couleur plus foncée.

Source : OCDE, Base de données PISA 2012, tableau I.2.1b.

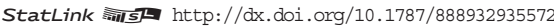

\section{L'amélioration de la performance} et le renforcement de l'équité sont

deux objectifs dont la réalisation

peut être concomitante.

Lorsque des pays voient leur performance s'améliorer, c'est généralement parce qu'ils sont parvenus à réduire leur pourcentage d'élèves peu performants. Ainsi, l'amélioration des résultats en compréhension de l'écrit de certains pays entre 2000 et 2009 s'explique par le recul du nombre de leurs élèves peu performants dans ce domaine. Ce constat vaut également pour l'amélioration des scores de certains pays en mathématiques entre 20003 et 2012, même si à Hong-Kong (Chine), en Italie, à Macao (Chine), en Pologne et au Portugal, I'amélioration de la performance en mathématiques au cours de cette période s'explique également par l'augmentation du nombre d'élèves très performants.

Comme de façon générale, dans tout effectif d'élèves, on compte plus d'éléments peu performants que d'éléments très performants, tout changement intervenant à ces deux extrémités du spectre de compétence tend à être plus important parmi les élèves peu performants que parmi leurs pairs plus performants. Ainsi, dans les pays et économies participant à l'enquête PISA, le pourcentage d'élèves très performants en mathématiques a évolué de 2.7 points de pourcentage, 
en moyenne, entre 20003 et 2012, tandis que celui des élèves peu performants dans ce domaine a évolué de 4.4 points de pourcentage au cours de cette période. De fait, seuls la Corée et Macao (Chine), deux systèmes d'éducation très performants, ont connu une augmentation sensible de leur pourcentage d'élèves très performants en mathématiques, sans pour autant enregistrer de recul de leur pourcentage d'élèves peu performants.

L'analyse tendancielle de la performance des pays dans les enquêtes PISA montre que le schéma d'évolution le plus répandu suit une courbe progressive et régulière. Le Brésil, pays qui a connu la plus forte amélioration parmi les participants à toutes les enquêtes PISA depuis 2003, n'a pas vu son score augmenter ou baisser de façon radicale pour atteindre sa bonne performance en mathématiques. Cette avancée relativement progressive vers l'excellence en mathématiques s'observe également en Allemagne, à Hong-Kong (Chine), en Pologne, en Tunisie et en Turquie.

\section{Évolution du pourcentage d'élèves peu ou très performants en mathématiques entre 2003 et 2012}

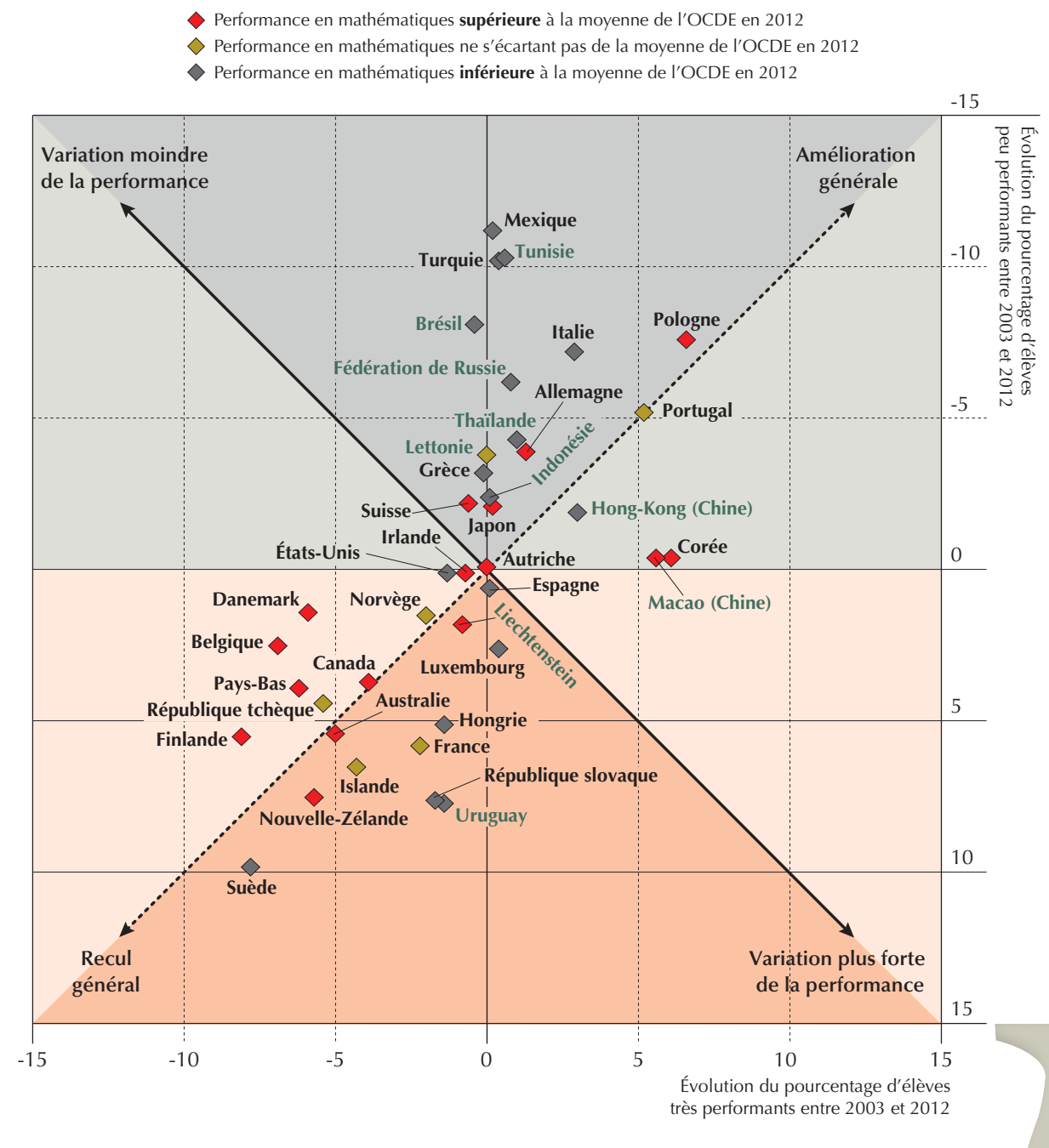

Remarque : ce graphique présente les pays/économies qui ont participé aux deux enquêtes PISA 2003 et PISA 2012. Source : OCDE, Base de données PISA 2012, tableau 1.2.1b.

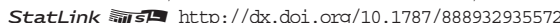


